\title{
Advanced Lemierre Syndrome Requiring Surgery
}

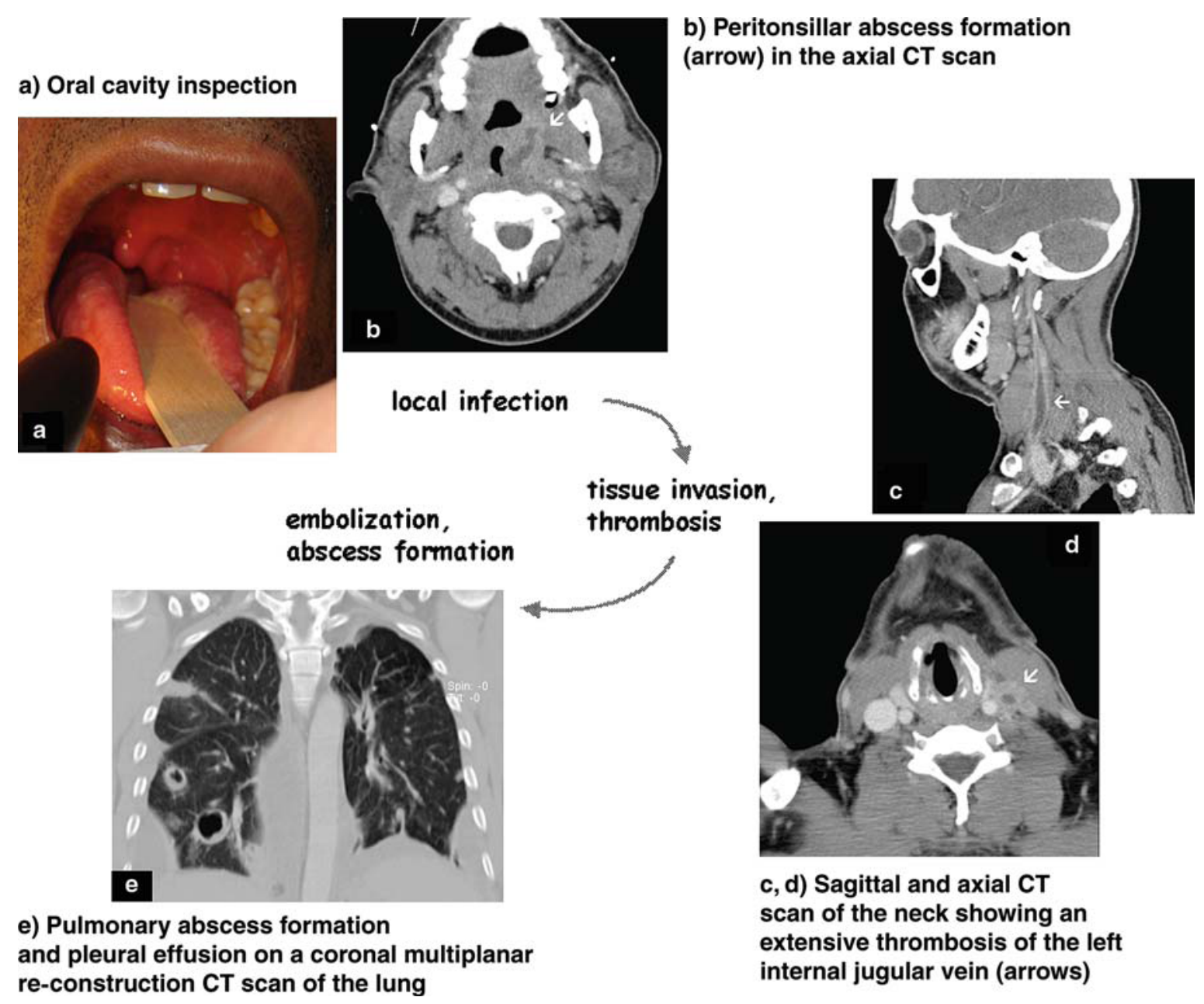

A 38-year-old homeless man was admitted with a 2-week history of a sore throat, increasing shortness of breath, and high fever. Clinical examination showed enlarged and tender submandibular and anterior cervical lymph nodes and a pronounced enlargement of the left peritonsillar region (Figure 1a). CT scan of the throat and the chest showed left peritonsillar abscess formation, occlusion of the left internal jugular vein with inflammatory wall thickening and perijugular soft tissue infiltration, pulmonary abscesses, and bilateral pleural effusions (Figures $1 \mathrm{~b}-\mathrm{e}$, arrowed). Anaerobe blood cultures grew Fusobacterium necrophorum, leading to the diagnosis of Lemierre's syndrome. Treatment with high-dose amoxicillin and clavulanic acid improved the oropharyngeal condition, but the patient's general status declined further, marked by dyspnea and tachypnea. Repeated CT scans showed progressive lung abscesses and bilateral

\author{
R. Escher \\ Division of Infectious Diseases, University Hospital, Bern, Switzerland \\ R. Escher (corresponding author), S. Haltmeier \\ Dept. of General Internal Medicine, University Hospital, Bern, 3010, \\ Switzerland; Phone: (+41/31) 632-4163, Fax: -8885 , \\ e-mail: robert.escher@insel.ch \\ N. von Steiger \\ Institute of Infectious Diseases, University Hospital, Bern, \\ Switzerland \\ A.E. Dutly \\ Dept. of General Thoracic Surgery, University Hospital, Bern, \\ Switzerland \\ A. Arnold \\ Dept. of ENT, Head and Neck Surgery, University Hospital, Bern, \\ Switzerland \\ R. Kickuth \\ Institute of Diagnostic, Interventional and Pediatric Radiology, \\ University Hospital, Bern, Switzerland \\ U. Schiemann \\ Dept. of General Internal Medicine, University Hospital, Bern, \\ Switzerland
}


pleural empyema. Bilateral tonsillectomy, ligation of the left internal jugular vein, and staged decortication of bilateral empyema were performed. Total antibiotic therapy duration was 9 weeks, including a change to peroral clindamycin. Clinical and laboratory findings had returned to normal 12 weeks after surgery.

The patient's history and the clinical and radiological findings are characteristic for Lemierre's syndrome. CT scans of the neck and the chest are the diagnostic methods of choice. F. necrophorum is found in over $80 \%$ of cases of Lemierre's syndrome and confirms the diagnosis. Prolonged antibiotic therapy is usually sufficient, but in se- lected patients, a surgical intervention may be necessary. Reported mortality rates are high, but in surviving patients, the recovery of pulmonary function is usually good.

R. Escher, S. Haltmeier, N. von Steiger, A.E. Dutly, A. Arnold, R. Kickuth, U. Schiemann

Infection 2008; 36: 495-496 DOI 10.1007/s15010-008-8216-7

Published online: September 13, 2008 